Research Article

\title{
The Study on Vibration Reduction of Nonlinear Time-Delay Dynamic Absorber under External Excitation
}

\author{
Tinghao Yan $\left(\mathbb{D},{ }^{1}\right.$ Chuanbo Ren $\left(\mathbb{D},{ }^{1}\right.$ Jilei Zhou $\mathbb{D}^{1},{ }^{1}$ and Sujuan Shao $\mathbb{D}^{2}$ \\ ${ }^{1}$ School of Traffic and Vehicle Engineering, Shandong University of Technology, Zibo 255000, China \\ ${ }^{2}$ School of Mechanical Engineering, Shandong University of Technology, Zibo 255000, China \\ Correspondence should be addressed to Chuanbo Ren; chuanbor@sdut.edu.cn
}

Received 23 August 2019; Revised 16 January 2020; Accepted 17 February 2020; Published 13 March 2020

Academic Editor: Rafael Morales

Copyright (c) 2020 Tinghao Yan et al. This is an open access article distributed under the Creative Commons Attribution License, which permits unrestricted use, distribution, and reproduction in any medium, provided the original work is properly cited.

The nonlinear time-delay dynamic absorber has a good control effect on the main system under harmonic excitation, but the control effect on the main system under complicated excitation is not obvious. In the previous study, they do not have an effective method to obtain the time-delay control parameters under different external excitations. Because of the above problems, this paper proposed an innovative method to obtain the control parameters of time-delay dynamic absorbers. For the vibration system including the nonlinear time-delay dynamic absorber, the transient time-integration method was used to solve the nonlinear delay dynamic differential equation of the system under the specific excitation. Then, the quantitative relationship was established between the time-domain responses of the vibration system, the external excitation, and time-delay control parameters. The timedelay control parameters would be obtained when the time-domain response of the main system is minimized under specific excitation using an algorithm that continuously compares the objective function that is related to the time-domain response of the main system. This paper took the two-degree-of-freedom vibration system with a nonlinear time-delay dynamic absorber as the target system to simulate the time-domain response of the main system. The simulation results show that the time-delay control parameters calculated by the new method can obtain significant vibration reduction effects for both harmonic excitation and multiharmonic excitation. The suppression performance of the nonlinear time-delay dynamic absorber is significantly improved.

\section{Introduction}

1.1. Background. The dynamic absorber is an effective vibration control tool, which has been widely used in the engineering industry, such as vehicles [1], ships, aerospace, and other fields [2]. The traditional passive dynamic vibration absorber has a small frequency reduction domain. Therefore, many scholars choose active control methods to improve the vibration reduction performance of the absorber. Time delay is an inevitable factor during the active control. So, many researchers focus on how to avoid and even use time delay as an active control factor.

1.2. The Problem Studied in this Paper. The time-delay absorber has a good control effect on the single-frequency excitation. However, the control effect of the constant timedelay parameters is not obvious when the external excitation is not harmonic excitation. Because the existing research does not have a good solution for the time-delay control parameters of the time-delay absorber under complicated excitation, for the above problems, this paper proposed an innovative method to calculate the control parameters of nonlinear time-delay absorber under multiharmonic external excitation.

1.3. Literature Survey. Roberson found that the nonlinear absorber can achieve better vibration absorption effects than the linear absorber [3]. Viguié and Kerschen addressed the problem of mitigating the vibration of nonlinear mechanical systems using nonlinear dynamical absorbers. It is effective in a wide range of forcing amplitudes [4]. Starosvetsky and Gendelman presented a nonlinear absorber tuning procedure in the vicinity of $(1: 1)$ resonance which provides the best total system energy suppression [5]. To further improve 
the vibration reduction performance of the absorber, the researchers chose to apply active control on it, such as choosing variable-stiffness structure [6] and controlling by adjusting the excitation current [7]. Oueini et al. proposed a nonlinear active vibration absorber for flexible structures and verified the effectiveness of the theoretically analyzed active vibration absorber by experiments [8]. Pappalardo and Guida proposed a new actively controlled inertial-based vibration absorber and used for controlling the mechanical deformations of flexible structures [9]. Also, some other ways to improve the bandwidth of passive absorbers were studied including the increase of the attached mass [10], the use of multiple (spread) tuned mass dampers [11], the addition of inerter devices [12], and the use of nonlinear dampers [13].

The active control system takes a certain time from the acquisition of the signal to the processing of the computer to the work of the actuator, so there is an inevitable time delay when the active control is performed [14]. The time delay can destabilize the stability or make it become nonlinear of the vibration system [15]. Therefore, researchers always treated the time delay as an unfavorable factor in early time. They usually excluded [16] and compensated [17] time delay using Pade approximation [18], sliding mode control [19], and other methods in engineering [20,21]. Olgac et al. [22-24] proposed to use time delay as the active control term to reduce the vibration by calculating the appropriate control parameters. The vibration of the main system under single-frequency excitation can be almost eliminated. The team studied the vibration reduction principle of the real-time tunable absorber and optimized the performance of the time-delay dynamic absorber.

In recent years, the research that uses time delay as an active vibration control term has made great progress [25-28]. Saeed et al. studied a controller based on nonlinear time-delay saturation to suppress the vibration of the nonlinear beam [29]. Alhazza et al. studied the nonlinear free vibration of beams using the acceleration time-delay feedback control [30] and studied the nonlinear vibration of the cantilever beam controlled by nonlinear time-delay feedback under parameter excitation [31] and experimented active-multimode vibration of the cantilever beam under the time-delay control [32]. Mirafzal et al. optimized the timedelay control parameters of the active vibration control of the cantilever beam by the genetic algorithm and carried out related experimental research, which showed that the method can achieve good results [33].

Zhao and $\mathrm{Xu}$ used the multiscale method to study the suppression performance of the nonlinear dynamic absorber with time delay for the main system [34-36]. Wang and $\mathrm{Hu}$ [37] did theoretical and methodological study on the stability of time-delay dynamic systems and the Hopf bifurcation behavior. Chen and Cai studied the active control on the flexible beam rotating vibration and forced vibration with time delay and carried out experimental research about it $[38,39]$. Li et al. studied the control method of varyingtime-delay for the vibration system under the action of multiharmonic excitation [40] and white noise excitation [41], and it has achieved a certain effect for the vibration control of the main system.
1.4. Scope and Contribution of this Study. In this paper, the two-degree-of-freedom vibration system with the nonlinear time-delay absorber is taken as an example to calculate the optimal time-delay control parameters under different external excitations. By analyzing the vibration response of the main system, the nonlinear constant time-delay absorber calculated in this paper has a very significant control effect for both harmonic excitation and multiharmonic excitation.

1.5. Organization of the Paper. In Section 2, the nonlinear time-delay absorber mechanical model is presented in Section 2.1, and the stability analysis of the main system attached with the nonlinear time-delay absorber is done in Section 2.2. Section 3 is the solving algorithm of the optimal time-delay control parameter. In Section 3.1, the numerical solution method for the system equations of this paper is proposed briefly. The relations of objective function and the responses of the main system in the time domain are illustrated in Section 3.2. In Section 3.3, the algorithm flow about how to get optimal control parameters is proposed. In Section 4, the computational simulation and result analysis about the main system controlled by the time-delay control parameters calculated in this paper is presented under harmonic excitation and multiharmonic excitation. Section 5 is a summary of the paper, including some general considerations on the work done, a short discussion on the quality of the numerical results found, and possible directions for future research.

\section{Nonlinear Time-Delay Absorber Model and Stability Analysis}

2.1. Mechanical Model. In practical engineering, the shape and structure of the dynamic absorber are complicated and contain amount of uncertainty. We made a reasonable simplification of the practical dynamic absorber for the study. A two-degree-of-freedom vibration system model including a nonlinear time-delay absorber is established. The model is shown in Figure 1.

The system includes a nonlinear time-delay absorber based on the displacement feedback of the absorber. The time-delay feedback control force is expressed as

$$
u=g x_{1}(t-\tau)
$$

In the system, $g$ is the feedback gain and $\tau$ is the time delay of control parameters. $m_{1}$ is the mass of the absorber, $k_{1}$ is the stiffness coefficient, and $c_{1}$ is the damping coefficient of the absorber, and $x_{1}$ is the vibration displacement of the absorber. $m_{2}$ is the mass of the main system, $k_{2}$ is the stiffness coefficient, and $c_{2}$ is the damping coefficient of the main system, and $x_{2}$ is the vibration displacement of the main system. $x_{d}$ is the external displacement excitation, and its form is harmonic or multiharmonic.

The nonlinear dynamic differential equation of the vibration system is expressed as 


$$
\left\{\begin{array}{l}
m_{1} \ddot{x}_{1}+k_{1}\left(x_{1}-x_{2}\right)+\mu k_{1}\left(x_{1}-x_{2}\right)^{3}+c_{1}\left(\dot{x}_{1}-\dot{x}_{2}\right)+u=0, \\
m_{2} \ddot{x}_{2}+k_{2}\left(x_{2}-x_{d}\right)+c_{2}\left(\dot{x}_{2}-\dot{x}_{d}\right)+k_{1}\left(x_{2}-x_{1}\right)+c_{1}\left(\dot{x}_{2}-\dot{x}_{1}\right)+\mu k_{1}\left(x_{2}-x_{1}\right)^{3}-u=0 .
\end{array}\right.
$$

2.2. Stability Analysis. The stability analysis of the system ensures that the optimized time-delay control parameters satisfy the system stability conditions.

For system dynamics differential equation (2), when $x_{d}=0$, regardless of the parameters in the system, the equilibrium point $(0,0)$ is always the solution of equation (2). This paper mainly focuses on the system at the equilibrium point $(0,0)$. The stability is analyzed. Linearizing equation (1) at equilibrium point $(0,0)$, we can get the corresponding characteristic equation as

$$
G(s)=A(s)+g e^{-\tau s} P(s)=0 .
$$

That

$$
\begin{aligned}
& \mathbf{A}(s)=\left(m_{1} s^{2}+c_{1} s+k_{1}\right) P(s)+m_{1} s^{2}\left(c_{1} s+k_{1}\right), \\
& P(s)=m_{2} s^{2}+c_{2} s+k_{2} .
\end{aligned}
$$

In system characteristic equation (3), the relationship between the gain and the time delay is

$$
|g|=\left(\frac{|A|}{|P|}\right) e^{\tau b}
$$

where $b$ is the real part of the characteristic equation root $s$ :

$$
\begin{aligned}
b & =\mathfrak{R}(s), \\
\angle P-\angle A & =(2 k+1) \pi+\tau \omega, \quad g>0, \\
\angle P-\angle A & =2 k \pi+\tau \omega, \quad g<0 .
\end{aligned}
$$

Among them, $k=0, \pm 1, \pm 2, \pm 3 \ldots$

It can be seen from equation (5) that the real part $b$ of the characteristic equation root $s$ increases as the absolute value of the time-delay gain $g$ increases. In another word, the characteristic root moves from the left half plane to the right half plane as $|g|$ becomes larger in the complex plane. The system switches from a steady state to an unstable state.

When the root of the characteristic equation is a pure virtual root $s= \pm \omega_{\mathrm{c}} \mathrm{i}$, the system is in a critical stable state, and equation (5) becomes

$$
|g|_{s=\omega_{c} i}=\frac{|A|}{|P|}
$$

For the specific time delay $\tau=\tau_{0}$, the system's crossing frequency is determined by function (7), and the corresponding critical stable gain can be determined by function (8). When the subscript $k=1,2,3, \ldots$ changes, the system's crossing frequency and critical gain change accordingly. In order to ensure that the system always remains stable, when the gain is greater than zero, the gain should be less than the minimum value of the critical stability gain of all systems corresponding to the time delay; when the gain is less than zero, the gain should be greater than the maximum value of the critical stability gain of all systems corresponding to the time delay.

$$
\begin{array}{ll}
g<g_{\omega c \min }\left(\tau\left(\omega_{c}\right)\right), & g>0, \\
g>g_{\omega c \max }\left(\tau\left(\omega_{c}\right)\right), & g<0 .
\end{array}
$$

Finally, the form of obtained time-delay feedback gain is an open interval where the minimum value is less than 0 and the maximum value is greater than 0 .

\section{Optimal Time-Delay Control Parameter Solving Method}

3.1. Numerical Solution Method for Nonlinear Differential Equations with Time Delay. Let equation (2)

$$
\begin{aligned}
& y_{1}=x_{1}, \\
& y_{2}=\dot{x}_{1}, \\
& y_{3}=x_{2}, \\
& y_{4}=\dot{x}_{2} .
\end{aligned}
$$

Write equation (2) as a state equation form:

$$
y^{\prime}(t)=\mathbf{A} y(t)+\mathbf{G} y_{1}(t-\tau)+\mathbf{B}\left(y_{1}(t)-y_{3}(t)\right)^{3}+\mathbf{F}(t)
$$

That

$$
\begin{aligned}
y(t) & =\left[\begin{array}{llll}
y_{1}(t) & y_{2}(t) & y_{3}(t) & y_{4}(t)
\end{array}\right]^{T}, \\
y^{\prime}(t) & =\left[\begin{array}{llll}
\dot{y}_{1}(t) & \dot{y}_{2}(t) & \dot{y}_{3}(t) & \dot{y}_{4}(t)
\end{array}\right]^{T},
\end{aligned}
$$

$$
\mathbf{A}=\left[\begin{array}{cccc}
0 & 1 & 0 & 0 \\
-\frac{k_{1}}{m_{1}} & -\frac{c_{1}}{m_{1}} & \frac{k_{1}}{m_{1}} & \frac{c_{1}}{m_{1}} \\
0 & 0 & 0 & 1 \\
\frac{k_{1}}{m_{2}} & \frac{c_{1}}{m_{2}} & -\frac{k_{1}+k_{2}}{m_{2}} & -\frac{c_{1}+c_{2}}{m_{2}}
\end{array}\right]
$$

$$
\mathbf{G}=\left[\begin{array}{llll}
0 & -\frac{g}{m_{1}} & 0 & \frac{g}{m_{2}}
\end{array}\right]^{T}
$$

$$
\mathbf{B}=\left[\begin{array}{llll}
0 & -\frac{\mu}{m_{1}} & 0 & \frac{\mu}{m_{2}}
\end{array}\right]^{T}
$$

$$
\mathbf{F}(t)=\left[\begin{array}{llll}
0 & 0 & 0 & \frac{c_{2}}{m_{2}} \dot{x}_{d}+\frac{k_{2}}{m_{2}} x_{d}
\end{array}\right]^{T}
$$

The delay feedback term, the nonlinear term, and the external excitation term in equation (11) are regarded as nonhomogeneous terms, and then the solution of the equation can be written as 


$$
\begin{aligned}
y(t)= & \exp (\mathbf{A} t) y(0)+\int_{0}^{t} \exp [\mathbf{A}(t-s)] \mathbf{G} y_{1}(s-\tau) \mathrm{d} s \\
& +\int_{0}^{t} \exp [\mathbf{A}(t-s)] \mathbf{B}\left(y_{1}(s)-y_{3}(s)\right)^{3} \mathrm{~d} s \\
& +\int_{0}^{t} \exp [\mathbf{A}(t-s)] \mathbf{F}(s) \mathrm{d} s .
\end{aligned}
$$

Equation (13) was numerically discretized. The step is $\Delta t=t_{k+1}-t_{k}$. If the simulation duration is $T$, the total number of steps is $m=T / \Delta t$, and a series of time points of equal steps $\Delta t$ is

$$
t_{0}=0, t_{1}=\Delta t, \ldots, t_{k}=k \times \Delta t, \quad k \in[0, m] .
$$

Then, the solution of the equation can be discretized into the following stepwise integral function of the recursive form

$$
\begin{aligned}
y_{k+1}= & \exp \left(\mathbf{A} \Delta_{t}\right) y_{k}+\int_{t_{k}}^{t_{k+1}} \exp \left[\mathbf{A}\left(t_{k+1}-s\right)\right] \mathbf{G} y_{1}(s-\tau) \mathrm{d} s \\
& \cdot \int_{t_{k}}^{t_{k+1}} \exp \left[\mathbf{A}\left(t_{k+1}-s\right)\right] \mathbf{B}\left(y_{1}(s)-y_{3}(s)\right)^{3} \mathrm{~d} s \\
& +\int_{t_{k}}^{t_{k+1}} \exp \left[\mathbf{A}\left(t_{k+1}-s\right)\right] \mathbf{F}(s) \mathrm{d} s .
\end{aligned}
$$

Then, the above function could be written as

$$
\begin{aligned}
y_{k+1}= & \exp \left(\mathbf{A} \Delta_{t}\right) y_{k}+\int_{k \Delta_{t}}^{(k+1) \Delta_{t}} \exp \left[\mathbf{A}\left[(k+1) \Delta_{t}-s\right]\right] \mathbf{F}(s) \mathrm{d} s \\
& +\int_{(k-m) \Delta_{t}}^{[(k+1)-m] \Delta_{t}} \exp \left[\mathbf{A}\left[(k+1) \Delta_{t}-s-m \Delta_{t}\right]\right] \mathbf{G} y_{1}(s) \mathrm{d} s \\
& +\int_{k \Delta_{t}}^{(k+1) \Delta_{t}} \exp \left[\mathbf{A}\left[(k+1) \Delta_{t}-s\right]\right] \mathbf{B}\left(y_{1}(s)-y_{3}(s)\right)^{3} \mathrm{~d} s .
\end{aligned}
$$

Among the above function, $m=(\tau / \Delta t)$. The initial condition of the function is

$$
y_{0}=\left[\begin{array}{llll}
0 & 0 & 0 & 0
\end{array}\right]^{T} \text {. }
$$

The above equation was solved by the transient timeintegration method. The specific solution method is discussed in another paper. The system vibration response in the time domain could be expressed as

$$
\left\{\begin{array}{l}
x_{1}=\left[x_{11}, x_{12} \ldots x_{1 k} \ldots x_{1 m}\right] \\
\dot{x}_{1}=\left[\dot{x}_{11}, \dot{x}_{12} \ldots \dot{x}_{1 k} \ldots \dot{x}_{1 m}\right], \\
x_{2}=\left[x_{21}, x_{22} \ldots x_{2 k} \ldots x_{2 m}\right] \\
\dot{x}_{2}=\left[\dot{x}_{21}, \dot{x}_{22} \ldots \dot{x}_{2 k} \ldots \dot{x}_{2 m}\right]
\end{array} \quad k \in[1, m]\right.
$$

$x_{1 k}$ is the vibration displacement, and $\dot{x}_{1 k}$ is the vibration velocity at the time $t_{k}$ of the mass $m_{1} . x_{2 k}$ is the vibration displacement, and $\dot{x}_{2 k}$ is the vibration velocity at the time $t_{k}$ of the mass $m_{2}$.

Function (2) was transformed as

$$
\left\{\begin{array}{l}
\ddot{x}_{1}=-\frac{k_{1}}{m_{1}} x_{1}+\frac{k_{1}}{m_{1}} x_{2}-\frac{c_{1}}{m_{1}} \dot{x}_{1}+\frac{c_{1}}{m_{1}} \dot{x}_{2}-\frac{\mu}{m_{1}}\left(x_{1}-x_{2}\right)^{3}-\frac{g}{m_{1}} x_{1}(t-\tau), \\
\ddot{x}_{2}=\frac{k_{1}}{m_{2}} x_{1}+\frac{c_{1}}{m_{2}} \dot{x}_{1}-\frac{k_{1}+k_{2}}{m_{2}} x_{2}-\frac{c_{1}+c_{2}}{m_{2}} \dot{x}_{2}+\frac{\mu}{m_{2}}\left(x_{1}-x_{2}\right)^{3}+\frac{g}{m_{2}} x_{1}(t-\tau)+\frac{k_{2}}{m_{2}} x_{d}+\frac{c_{2}}{m_{2}} \dot{x}_{d} .
\end{array}\right.
$$

The result of function (18) $x_{1 k}, \dot{x}_{1 k}, x_{2 k}, \dot{x}_{2 k}$ was taken into function (19) to obtain the acceleration $\ddot{x}_{1 k}$ of the mass $m_{1}$ at time $t_{k}$ and the acceleration $\ddot{x}_{2 k}$ of the mass $m_{2}$ at time $t_{k}$.
3.2. Objective Function. The time-domain vibration response of the main system was directly used as the objective function to optimize the time-delay control parameters with the control effect. This paper chose the weighted root-mean- 


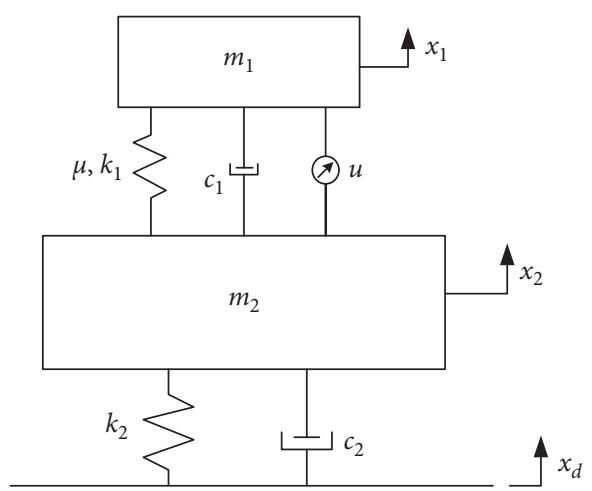

Figure 1: Two-degree-of-freedom vibration system with the nonlinear time-delay absorber.

square of the main system's time-domain vibration response as the objective function.

$$
\begin{aligned}
& \text { Min } J=c_{1} J_{d}+c_{2} J_{v}+c_{3} J_{a} \\
& \text { s.t. }\left\{\begin{array}{l}
0<\tau<20, \\
g \in\left(g_{\min }, g_{\max }\right),
\end{array}\right. \\
& J_{d}=\sqrt{\frac{\sum_{k=1}^{n}\left(x_{1 k}\right)^{2}}{n},} \\
& J_{v}=\sqrt{\frac{\sum_{k=1}^{n}\left(\dot{x}_{1 k}\right)^{2}}{n},} \\
& J_{a}=\sqrt{\frac{\sum_{k=1}^{n}\left(\ddot{x}_{1 k}\right)^{2}}{n}},
\end{aligned}
$$

where $J_{d}$ is the root-mean-square of the displacement, $J_{v}$ is the root-mean-square of the velocity, and $J_{a}$ is the rootmean-square of the acceleration. $c_{1}, c_{2}$, and $c_{3}$ are weight coefficients, which can be selected according to the requirements of the time-delay dynamic absorber. In this paper, we chose it as $c_{1}=c_{2}=c_{3}=1$.

\subsection{Time-Delay Control Parameter Solving Algorithm.} The algorithm randomly gave a set of initial control parameters of the time-delay absorber within the stability interval (20) to ensure the initial stability of the vibration system.

The numerical solution of system dynamics equation (2) is solved by the transient time-integration method. We could get the vibration displacement, velocity, and acceleration of the main system corresponding to each time $t_{k}$ under the initial control parameters. According to objective function (23), the objective function value $J$ under each pair of timedelay control parameters is obtained. Then, the set of control parameters would be updated, and equation (2) controlled by the new parameters is solved again. The new objective function value $J$ is calculated. By continuously updating the control parameters $(\tau, g)$ and comparing the objective function value $J$, we select the control parameters when the objective function $J$ is the smallest as the optimal control parameters.

The flowchart of the optimization algorithm is shown in Figure 2.

In the first round of calculation, firstly, we selected randomly $n$ pairs of time-delay control parameters $\left(\tau_{n}, g_{n}\right)$ in stable interval (20), which are recorded as the form array:

$$
\left\{\begin{array}{l}
\mathbf{T}_{1 \times n}^{1}=\left[\tau_{1}, \tau_{2}, \tau_{3} \ldots \tau_{n}\right], \\
\mathbf{G}_{1 \times n}^{1}=\left[g_{1}, g_{2}, g_{3} \ldots g_{n}\right] .
\end{array}\right.
$$

We can calculate the numerical solution of system dynamics equation (2) under the action of each pair of timedelay control parameters $\left(\tau_{n}, g_{n}\right)$ through the transient time-integration method. According to objective function (23), we calculate the corresponding main system displacement root-mean-square $J_{d}$, velocity root-mean-square $J_{v}$, acceleration root-mean-square $J_{a}$, and objective function $J$, and then store it with an array recorded as

$$
\mathbf{J}_{1 \times n}^{1}=\left[J_{1}, J_{2}, \ldots, J_{n}\right]
$$

$m$ is the number of rounds which the objective function is located, and $n$ is the position of the objective function in each array. The evaluation indexes $J_{1}$ to $J_{n}$ are compared one by one in the array. The time-delay control parameters $\tau$ and $g$ corresponding to the minimum evaluation index are selected as the optimal values $\tau_{p}^{1}$ and $g_{p}^{1}$ of the group. The smallest evaluation index $J_{\min }$ in the array is recorded as $J_{p}^{1}$. When calculating the first round, the global optimal evaluation index $J_{g}$ is equal to the first group optimal evaluation index, and the global optimal values $\tau_{g}$ and $g_{g}$ are equal to the first group optimal values $\tau_{p}^{1}$ and $g_{p}^{1}$.

After obtaining the optimal time-delay control parameters in the first group, the time-delay control parameters are updated, and the update rules are as follows:

$$
\left\{\begin{array}{l}
\tau_{(m, n)}=\left|w \tau_{(m-1, n)}+a_{1} r_{1}\left(\tau_{p}^{m-1}-\tau_{(m-1, n)}\right)+a_{2} r_{2}\left(\tau_{g}-\tau_{(m-1, n)}\right)\right| \\
g_{(m, n)}=w g_{(m-1, n)}+a_{1} r_{1}\left(g_{p}^{m-1}-g_{(m-1, n)}\right)+a_{2} r_{2}\left(g_{g}-g_{(m-1, n)}\right)
\end{array}\right.
$$

where $m$ is the number of rounds which the control parameters have been updated and $n$ is the position of the control parameters in each group. $\tau_{p}^{m-1}$ and $g_{p}^{m-1}$ are the optimal control parameter values in the $m-1$ round, and $\tau_{g}$ and $g_{g}$ are global optimal values. The parameters in function (27) are selected as $w=0.6, a_{1}=1.2, a_{2}=2.2$, and $r_{1}$ and $r_{2}$ are random numbers uniformly distributed between 0 and 1 . Since the time delay $\tau>0$ is meaningful, the time-delay $\tau$ update 


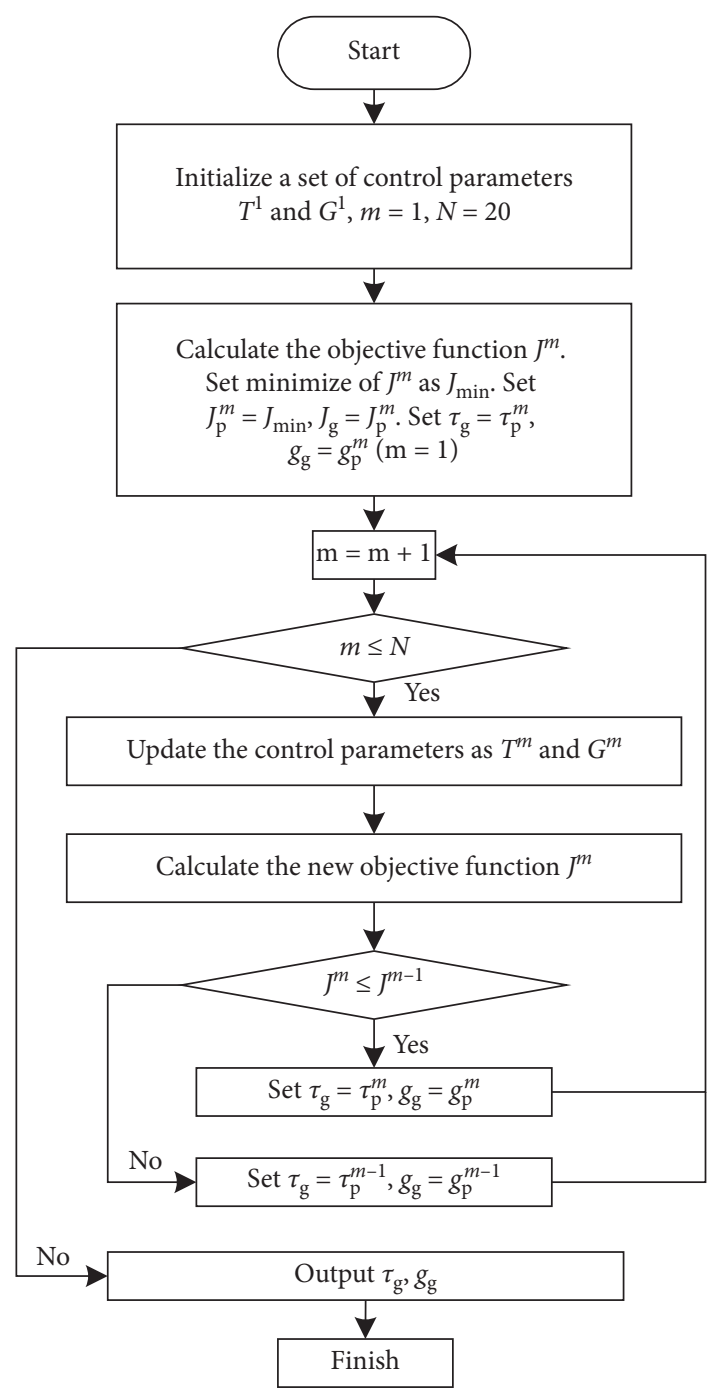

FIgURE 2: Flowchart of the optimization algorithm.

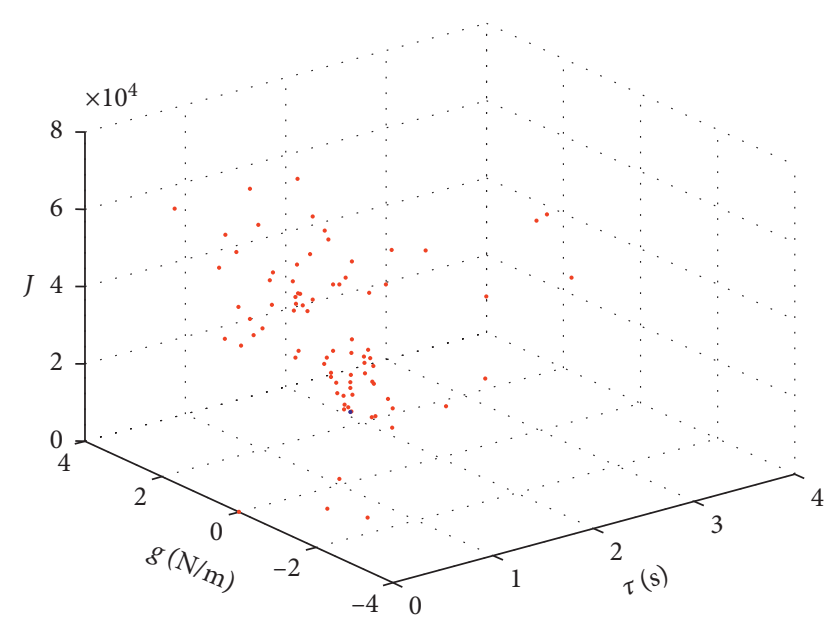

FIgURE 3: The relationship between the control parameters and the objective function.

function adds the absolute value sign. After updating, the next set of control parameters can be obtained. The $n$-th time-delay control parameter of the $m$-th round is expressed as

$$
\left\{\begin{array}{l}
\mathbf{T}_{1 \times n}^{m}=\left[\tau_{1}, \tau_{2}, \tau_{3} \ldots \tau_{n}\right], \\
\mathbf{G}_{1 \times n}^{m}=\left[g_{1}, g_{2}, g_{3} \ldots g_{n}\right] .
\end{array}\right.
$$

When the second round of $m=2$ is calculated, the second set of calculated objective functions is stored according to the system dynamics equation solving method and the objective function calculation method in the first round.

$$
\mathbf{J}_{1 \times n}^{m}=\left[J_{1}, J_{2}, \ldots, J_{n}\right]
$$

The optimal time-delay control parameters corresponding to the minimum objective function $J_{p}^{m}$ in the second group are recorded as $\tau_{p}^{m}$ and $g_{p}^{m}$.

The minimum evaluation index of the $m=2$ round is compared with the global minimum evaluation index. If the minimum evaluation index in the round is smaller than the global minimum evaluation index $\left(J_{p}^{m}<J_{q}\right)$, the optimal control parameters in the round is used as the global optimal control parameter $\left(\tau_{g}=\tau_{p}^{m}, g_{g}=g_{p}^{m}\right)$; if the minimum evaluation index in the round is not less than the global minimum evaluation index $\left(J_{p}^{m} \geq J_{g}\right)$, the global optimal control parameter is unchanged $\left(\tau_{g}=\tau_{g}, g_{g}=g_{g}\right)$.

The control parameters are updated again, and then the third round would be calculated. Repeat the above steps to calculate $N$ rounds. The global optimal control parameters are continuously updated, and the global optimal control parameters $\tau_{g}$ and $g_{g}$ at the end of the calculation are regarded as the optimal time-delay control parameters.

\section{Computational Simulation and Result Analysis}

4.1. Harmonic Excitation. When the external excitation is the harmonic form, the external excitation $x_{d}$ in function (2) can be expressed as

$$
x_{d}=a \sin (\omega t)
$$

In this paper, the parameters of the system are $m_{1}=0.1 \mathrm{~kg}, \quad m_{2}=1 \mathrm{~kg}, \quad k_{1}=10 \mathrm{~N} / \mathrm{m}, \quad k_{2}=100 \mathrm{~N} / \mathrm{m}$, $c_{1}=0.1 \mathrm{~N} * \mathrm{~s} / \mathrm{m}, c_{2}=2 \mathrm{~N} * \mathrm{~s} / \mathrm{m}, a=0.06 \mathrm{~m}$, and $\omega=10 \mathrm{rad} / \mathrm{s}$.

When the nonlinear coefficient is $\mu=0.5$ of the nonlinear absorber, the time-delay control parameter is $\tau=1.1924 \mathrm{~s}$, $g=-1.5742 \mathrm{~N} / \mathrm{m}$ optimized by the above algorithm. According to the stability analysis method, when the time delay is $\tau=1.1924 \mathrm{~s}$, the stable interval of the gain $g$ is $-10 \mathrm{~N} /$ $\mathrm{m}, 9.22 \mathrm{~N} / \mathrm{m}$, and the optimized result satisfies the stability condition.

The relationship between the control parameters and the objective function during the optimization process is shown in Figure 3. It can be seen from Figure 3 that the optimization result has a trend of convergence to the optimal control parameters.

The passive absorber with the same nonlinear coefficient was elected as the comparison. There are the time-domain simulation results of the main system with two different kinds of absorber.

It can be seen from the time-domain simulation results, Figures 4-6, that the nonlinear time-delay dynamic absorber 


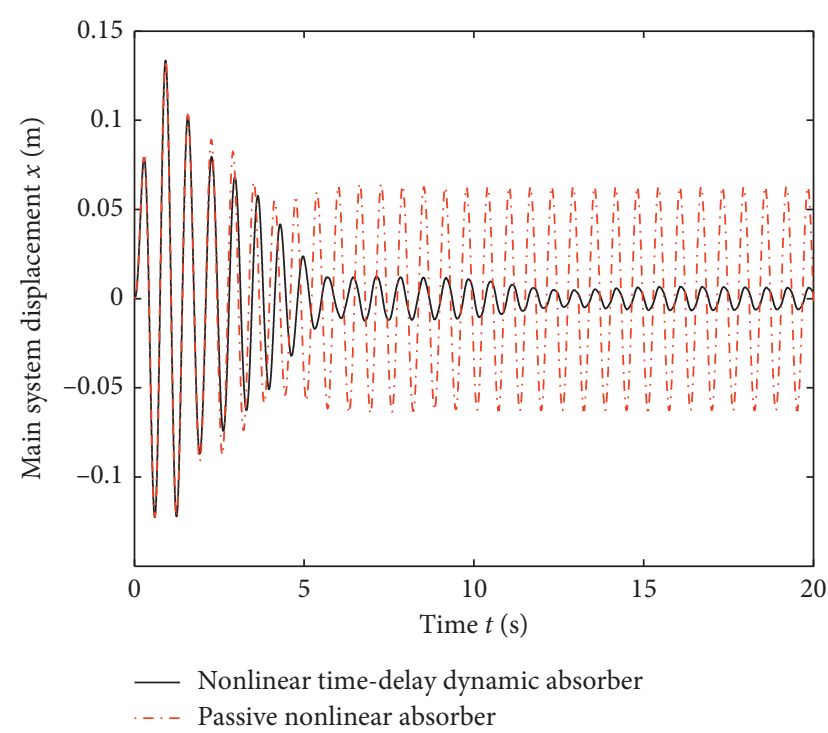

FIgUre 4: Displacement of the main system.

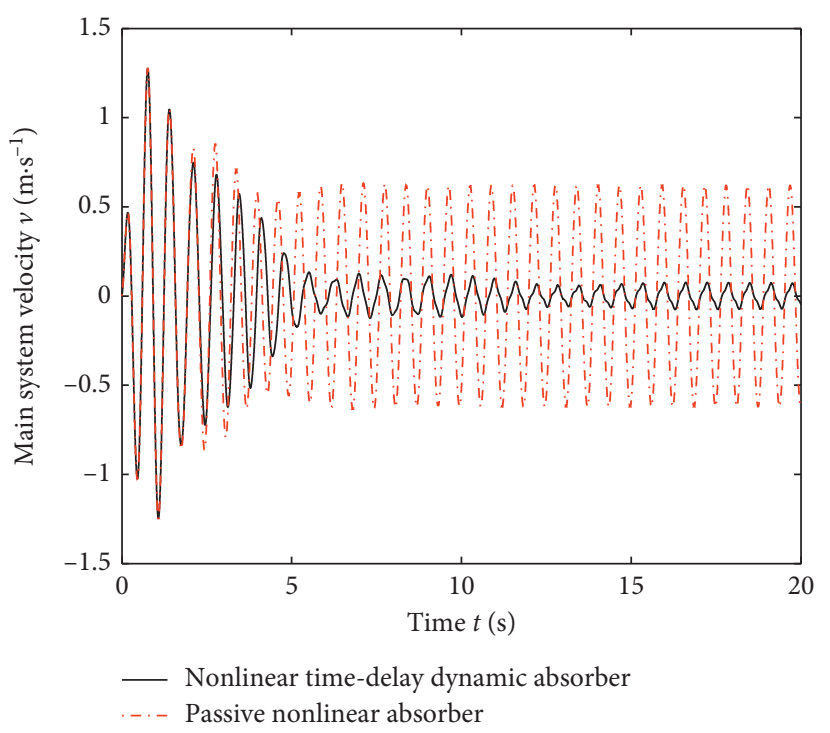

FIgURE 5: Velocity of the main system.

can achieve a better control effect than the nonlinear passive absorber. After the nonlinear time-delay dynamic absorber worked, the vibration response of the main system under the harmonic excitation was reduced more than $80 \%$. It can be seen from the phase diagram (Figure 7) of the main system and (Figure 8) of the absorber that both the main system and the absorber are stable when the nonlinear time-delay dynamic absorber is working. Under the action of harmonic excitation, the algorithm can obtain suitable time-delay control parameters to effectively control the vibration of the main system, which verifies the effectiveness of the nonlinear time-delay dynamic absorber in previous studies.

4.2. Multiharmonic Excitation. It is well known that complicated excitation can be represented by a superposition of harmonic excitation. In this paper, we choose to construct a

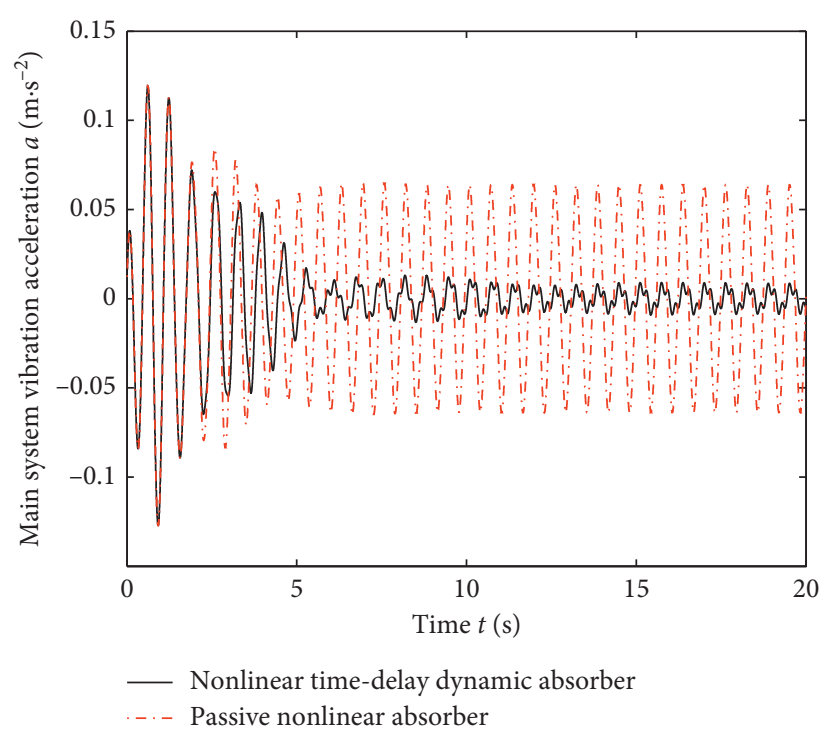

Figure 6: Acceleration of the main system.

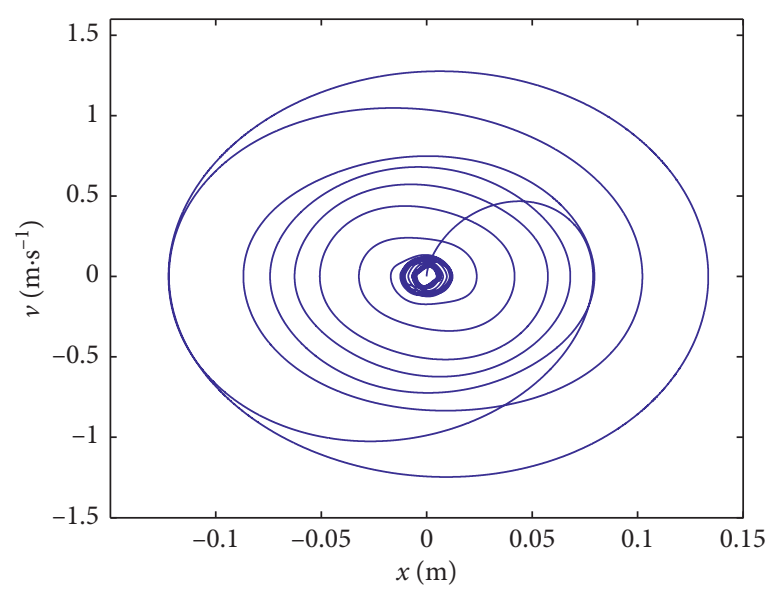

_ Main system phase diagram

Figure 7: The phase diagram of the main system.

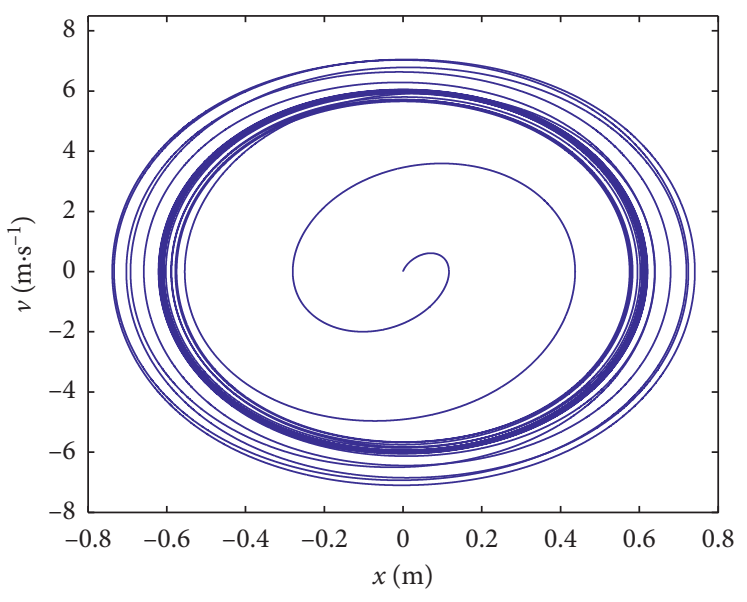

_ Absorber phase diagram

Figure 8: The phase diagram of the absorber. 


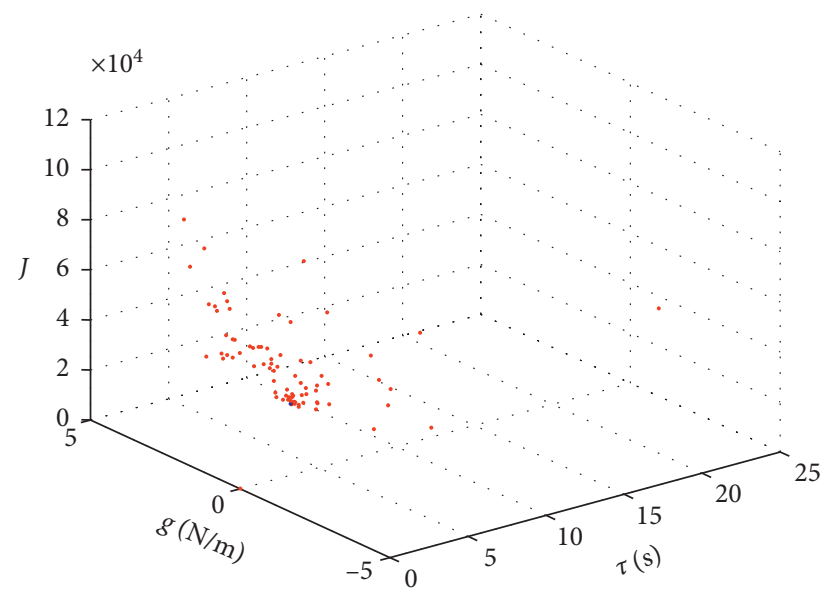

FIGURE 9: The relationship between the control parameters and the objective function.

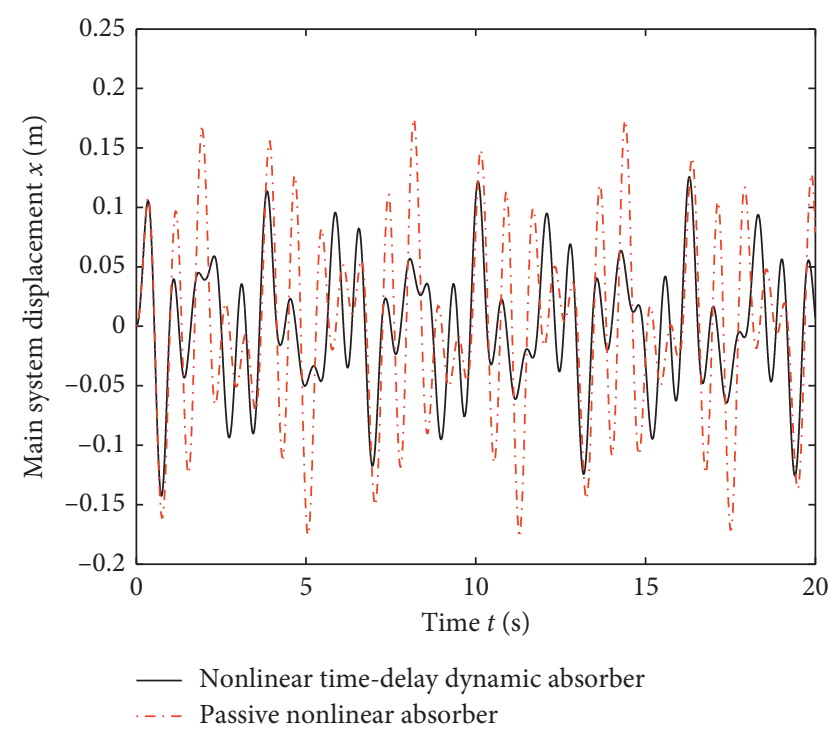

Figure 10: Displacement of the main system.

complicated excitation by the following method to study the control effect of the nonlinear time-delay dynamic absorber on the main system under complicated excitation.

$$
\begin{gathered}
x_{d}=\sum_{i=1}^{\infty} \xi_{i}(t)=\sum_{i=1}^{\infty} \varphi_{i} \sin \left(\omega_{i} t+\theta_{i}\right) \\
\left\{\begin{array}{l}
\varphi_{1}=0.04 \mathrm{~m}, \varphi_{2}=0.03 \mathrm{~m}, \varphi_{3}=0.025 \mathrm{~m}, \\
\omega_{1}=3.071 \mathrm{rad} / \mathrm{s}, \omega_{2}=7.071 \mathrm{rad} / \mathrm{s}, \omega_{3}=9.071 \mathrm{rad} / \mathrm{s}, \\
\theta_{1}=1.8 \mathrm{rad}, \theta_{2}=0 \mathrm{rad}, \theta_{3}=-1.2 \mathrm{rad} .
\end{array}\right.
\end{gathered}
$$

The nonlinear coefficient of the absorber is chosen as $\mu=0.5$. Through the time-delay control parameter solving algorithm given above, it can be found that, under the multiharmonic excitation, the time-delay control parameters are $\tau=0.0034 \mathrm{~s}$ and $g=-6.0397 \mathrm{~N} / \mathrm{m}$. According to the stability analysis method, when the time delay is calculated

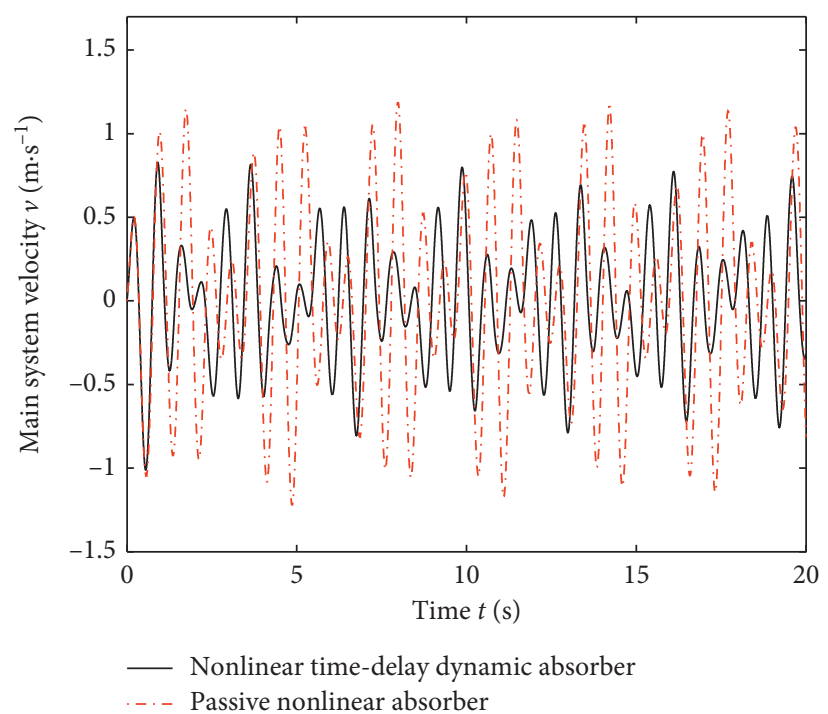

Figure 11: Velocity of the main system.

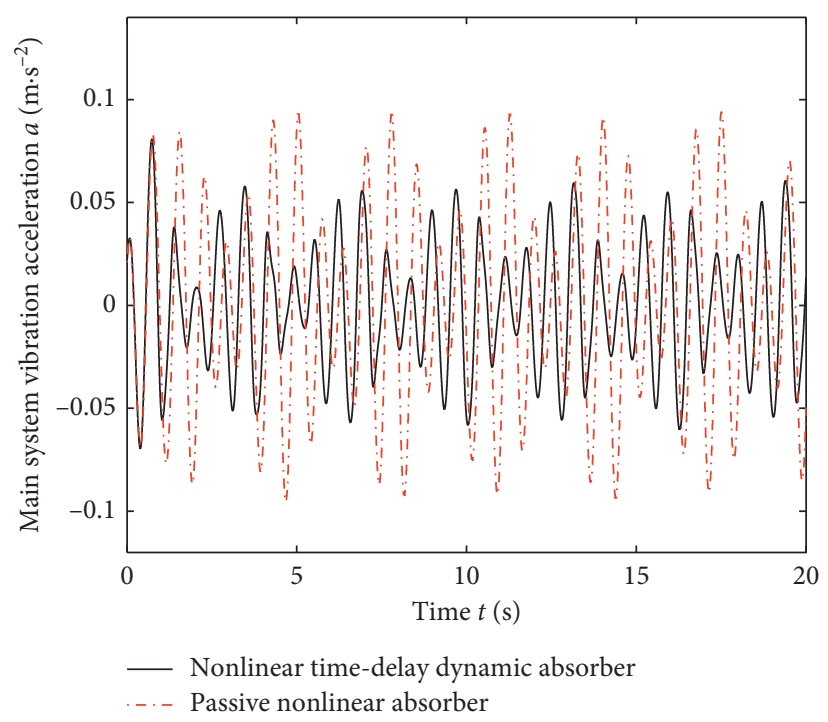

FIgURE 12: Acceleration of the main system.

as $\tau=0.0034 \mathrm{~s}$, the stability interval of the gain $g$ is $-10 \mathrm{~N} / \mathrm{m}$, $85431 \mathrm{~N} / \mathrm{m}$, and the optimized result satisfies the stability condition.

The relationship between the control parameters and the objective function during the optimization process is shown in Figure 9. It can be seen from Figure 9 that the optimization result has a trend of convergence to the optimal control parameters.

Figures 10-12 show the time-domain simulation results.

When the simulation duration is $300 \mathrm{~s}$, Figures 13 and 14 show the phase diagram of the absorber and the main system.

Under the action of the nonlinear time-delay dynamic vibration absorber, it can be seen from the time-domain simulation results, Figures $10-12$, that the vibration response of the main system is greatly attenuated under multiharmonic excitation. Observing the phase diagram, 


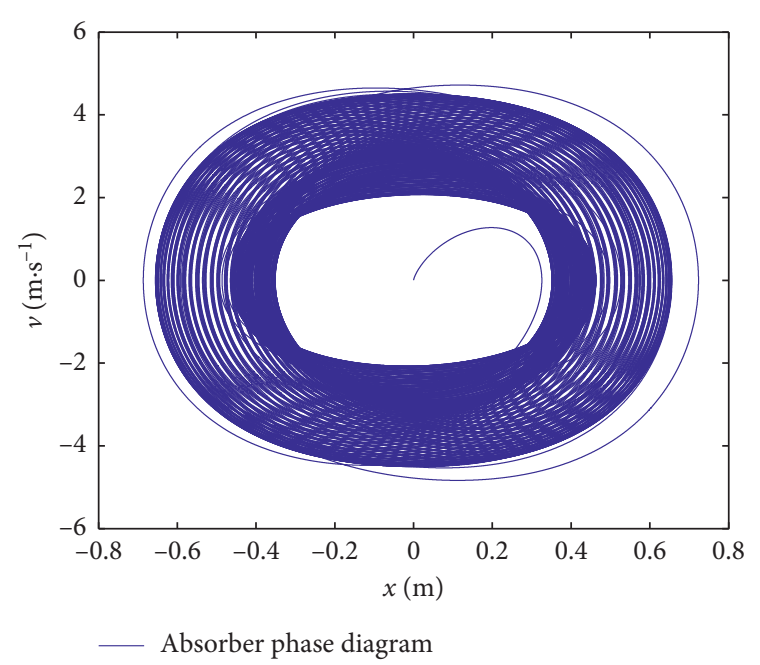

FIgURE 13: The phase diagram of the absorber.

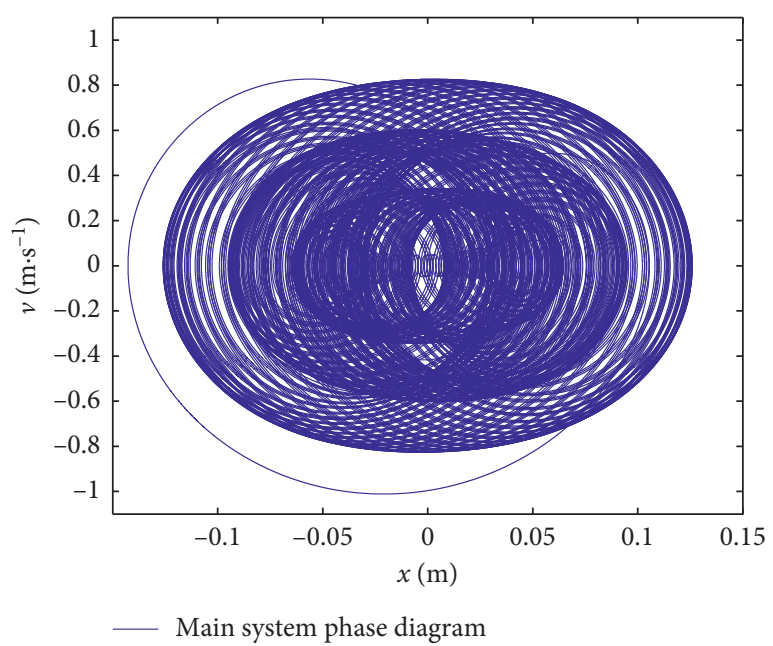

Figure 14: The phase diagram of the main system.

Figures 13 and 14, with simulation duration of 300 s, it can be seen that the main system and the absorber are in a stable state under the action of the nonlinear time-delay dynamic absorber. Compared with the nonlinear passive absorber, the vibration displacement response of the main system under the control of the nonlinear time-delay dynamic absorber can be attenuated by $33.92 \%$, and the vibration velocity response of the main system can be attenuated by $39.35 \%$. The system vibration acceleration can be attenuated by $38.6 \%$.

\section{Conclusion}

In this paper, a general and effective time-delay control parameter optimization solving method is proposed to improve the vibration reduction effect of the nonlinear timedelay dynamic absorber. The time-delay control parameters, external excitations, and the objective function were linked through the transient time-integration method. The minimum time-domain vibration response of the main system is defined as the optimization target, and the time-delay control parameters are directly optimized under specific external excitation. Whether it is harmonic excitation or multiharmonic excitation, the method can solve the appropriate time-delay control parameters to reduce the vibration of the main system.

The nonlinear time-delay dynamic absorber obtained through the method of this paper can effectively control the vibration response of the main system. The main conclusions are as follows:

(1) Whether it is simple harmonic excitation or multiharmonic excitation, this method can obtain suitable time-delay control parameters, which can effectively reduce the vibration response of the main system.

(2) The algorithm that takes the main system time-domain vibration response (displacement, velocity, and acceleration) as the objective function can directly obtain the time-delay control parameters that have control effect on the main system. The time-delay control parameters calculated by the algorithm are different under different external excitations.

(3) When the time-delay control parameters fluctuate within a small range around the optimal value, the time-delay dynamic vibration absorber also has an effect, which shows it has certain control robustness.

The transient time-integration method has high accuracy and good controllability when obtaining the numerical solutions of nonlinear time-delay dynamic problems. It is a good numerical method for studying nonlinear dynamics problems with time delay.

In the future, we will study the time-delay control on more complicated external excitation. The ideas in this paper can also be applied to the study of time-varying delay control, and it is also our direction in the future research.

\section{Data Availability}

The data used to support the findings of this study are included within the article. The results of this paper can be verified according to the data and methods given in the paper.

\section{Conflicts of Interest}

The authors declare that they have no conflicts of interest.

\section{Acknowledgments}

This study was supported with project no. 51275280 by the National Natural Science Foundation of China.

\section{References}

[1] G. Z. Liu, W. K. Shi, Y. S. Zheng et al., "Application of dynamic vibration absorbers in the vibration and noise reduction of drive axles," Journal of Vibration and Shock, vol. 37, no. 14, pp. 202-207, 2018. 
[2] H. Y. Hu, D. L. Guo, and J. S. Weng, "Recent advances in semi-active control of vibration," Journal of Vibration, Measurtment \& Diagnosis, vol. 21, no. 4, pp. 3-12, 2001.

[3] R. E. Roberson, "Synthesis of a nonlinear dynamic vibration absorber," Journal of the Franklin Institute, vol. 254, no. 3, pp. 205-220, 1952.

[4] R. Viguié and G. Kerschen, "Nonlinear vibration absorber coupled to a nonlinear primary system: a tuning methodology," Journal of Sound and Vibration, vol. 326, no. 3-5, pp. 780-793, 2009.

[5] Y. Starosvetsky and O. V. Gendelman, "Attractors of harmonically forced linear oscillator with attached nonlinear energy sink. II: optimization of a nonlinear vibration absorber," Nonlinear Dynamics, vol. 51, no. 1-2, pp. 47-57, 2007.

[6] M. X. Li and J. Liu, "Study on stability of the vibration control system with variable-stiffness structure," Journal of Vibration and Shock, vol. 18, no. 2, pp. 81-83, 1999.

[7] Z. Y. Hao, Z. P. Zeng, and G. Q. Shu, "Investigation on active torsional vibration absorber for engine crankshaft system," Journal of Vibration Engineering, vol. 7, no. 2, pp. 209-216, 1994.

[8] S. S. Oueini, A. H. Nayfeh, and J. R. Pratt, "A nonlinear vibration absorber for flexible structures," Nonlinear Dynamics, vol. 15, no. 3, pp. 259-282, 1998.

[9] C. M. Pappalardo and D. Guida, "Development of a new inertial-based vibration absorber for the active vibration control of flexible structures," Engineering Letters, vol. 26, no. 3, p. 11, 2018.

[10] M. De Angelis, S. Perno, and A. Reggio, "Dynamic response and optimal design of structures with large mass ratio TMD," Earthquake Engineering \& Structural Dynamics, vol. 41, no. 1, pp. 41-60, 2012.

[11] K. Xu and T. Igusa, "Dynamic characteristics of multiple substructures with closely spaced frequencies," Earthquake Engineering \& Structural Dynamics, vol. 21, no. 12, pp. 1059-1070, 1992.

[12] L. Marian and A. Giaralis, "Optimal design of a novel tuned mass-damper-inerter (TMDI) passive vibration control configuration for stochastically support-excited structural systems," Probabilistic Engineering Mechanics, vol. 38, pp. 156-164, 2014.

[13] F. Ricciardelli and B. J. Vickery, "Tuned vibration absorbers with dry friction damping," Earthquake Engineering \& Structural Dynamics, vol. 28, no. 7, pp. 707-723, 1999.

[14] J. Xu and L. J. Pei, "Advances in dynamics for delayed system," Advances in Mechanics, vol. 36, no. 1, pp. 17-30, 2006.

[15] H. Y. Hu and Z. H. Wang, "Review on nonlinear dynamics systems involving time delays," Advances in Mechanics, vol. 29, no. 4, pp. 501-512, 1999.

[16] S. Y. Chu, T. T. Soong, C. C. Lin, and Y. Z. Chen, "Time-delay effect and compensation on direct output feedback controlled mass damper systems," Earthquake Engineering \& Structural Dynamics, vol. 31, no. 1, pp. 121-137, 2002.

[17] H. Du, N. Zhang, and J. Lam, "Parameter-dependent inputdelayed control of uncertain vehicle suspensions," Journal of Sound and Vibration, vol. 317, no. 3-5, pp. 537-556, 2008.

[18] B. Ebrahimi, R. Tafreshi, M. Franchek, K. Grigoriadis, and J. Mohammadpour, "A dynamic feedback control strategy for control loops with time-varying delay," International Journal of Control, vol. 87, no. 5, pp. 887-897, 2014.

[19] F. Gouaisbaut, M. Dambrine, and J. P. Richard, "Robust control of delay systems: a sliding mode control design via LMI," Systems \& Control Letters, vol. 46, no. 4, pp. 219-230, 2002.
[20] H. Su and G. Y. Tang, "Vibration control for active suspention systems with time-delay input," Control Theory \& Application, vol. 33, no. 4, pp. 552-558, 2016.

[21] Z. Y. Wu, X. Zhou, D. W. Hu et al., "Active vibration suppression for boom of concrete pump truck based on timedelay compensation approach," China Mechanical Engineering, vol. 24, no. 24, pp. 3283-3288, 2013.

[22] N. Olgac, D. M. McFarland, and B. T. Holm-Hansen, "Position feedback-induced resonance: the delayed resonator," Winter Annual Meeting, vol. 38, pp. 113-119, 1992.

[23] N. Olgac and B. T. Holm-Hansen, "A novel active vibration absorption technique: delayed resonator," Journal of Sound and Vibration, vol. 176, no. 1, pp. 93-104, 1994.

[24] N. Olgac and B. Holm-Hansen, "Design considerations for delayed-resonator vibration absorbers," Journal of Engineering Mechanics, vol. 121, no. 1, pp. 80-89, 1995.

[25] C. Chen, J. Xiong, Z. Wan, J. Lei, and K. Zhang, “A Time delay compensation method based on area equivalence for active damping of an LCL-type converter," IEEE Transactions on Power Electronics, vol. 32, no. 1, pp. 762-772, 2017.

[26] G. Hailatig and Z. H. Hu, "Design of wide-area time-delay supplementary controller for interconnected network based on Hamilton function method," IOP Conference Series: Earth and Environmental Science, vol. 40, no. 1, pp. 012-044, 2016.

[27] N. Jalili, "A comparative study and analysis of semi-active vibration- control systems," Journal of Vibration and Acoustics, vol. 124, no. 4, pp. 593-605, 2002.

[28] L. R. C. Drehmer, W. J. P. Casas, and H. M. Gomes, "Parameters optimisation of a vehicle suspension system using a particle swarm optimisation algorithm," Journal of Vehicle System Dynamics, vol. 53, no. 4, pp. 449-474, 2015.

[29] N. A. Saeed, W. A. El-Ganini, and M. Eissa, "Nonlinear time delay saturation-based controller for suppression of nonlinear beam vibrations," Applied Mathematical Modelling, vol. 37, pp. 8846-8864, 2013.

[30] K. A. Alhazza, Z. N. Masoud, and M. Alajmi, "Nonlinear free vibration control of beams using acceleration delayed-feedback control," Smart Materials and Structures, vol. 17, no. 1, Article ID 015002, 2008.

[31] K. A. Alhazza, "Non-linear vibrations of parametrically excited cantilever beams subjected to non-linear delayedfeedback control," International Journal of Non-Linear Mechanics, vol. 43, pp. 801-812, 2008.

[32] K. A. Alhazza, H. Nayfeh, and M. F. Daqaq, "On utilizing delayed feedback for active-multimode vibration control of cantilever beams," Journal of Sound and Vibration, vol. 319, no. 3-5, pp. 735-752, 2009.

[33] S. H. Mirafzal, A. M. Khorasani, and A. H. Ghasemi, "Optimizing time delay feedback for active vibration control of a cantilever beam using a genetic algorithm," Journal of Vibration and Control, vol. 22, no. 19, pp. 4047-4061, 2016.

[34] Y. Y. Zhao and J. Xu, "Mechanism analysis of delayed nonlinear vibration absorber," Chinese Journal of Theoretical and Applied Mechanics, vol. 40, no. 1, pp. 98-106, 2008.

[35] Y. Y. Zhao and J. Xu, "Effects of delayed feedback control on vibration suppression in an auto-parametric dynamical absorber," Acta Mechanica Solida Sinic, vol. 28, no. 4, pp. 347-354, 2007.

[36] Y. Y. Zhao and J. Xu, "Effects of delayed feedback control on nonlinear vibration absorber system," Journal of Sound and Vibration, vol. 308, no. 1-2, pp. 212-230, 2007.

[37] Z. H. Wang and H. Y. Hu, "Stability and bifurcation of delayed dynamics system:from theory to application," Advances in Mechanics, vol. 43, no. 1, pp. 3-20, 2013. 
[38] L. X. Chen and G. P. Cai, "Experimental study on active control of a rotating flexible beam with time delay," Chinese Journal of Theoretical and Applied Mechanics, vol. 40, no. 4, pp. 520-527, 2008.

[39] L. X. Chen and G. P. Cai, "Experimental study of variable structure control in time-delay for a flexible beam under forced vibration," Chinese Journal of Theoretical and Applied Mechanics, vol. 41, no. 3, pp. 410-417, 2009.

[40] S. Li, J. L. Zhou, C. B. Ren et al., "Time varying delay feedback vibration control for multipled excitation forces," Journal of Guangxi University (Natural Science Edition), vol. 43, no. 3, pp. 982-993, 2018.

[41] S. Li, J. L. Zhou, C. B. Ren et al., "The research of time delay vibration control with time-varying parameters," Chinese Journal of Theoretical and Applied Mechanics, vol. 50, no. 1, pp. 99-108, 2018. 